\title{
Controllable Synthesis of Nitrogen-Doped Graphene Oxide by Tablet-Sintering for Efficient Lithium/Sodium-Ion Storage
}

\author{
Mingxiang Hu, ${ }^{1}$ Qian $\mathrm{Lv}^{1}$ and Ruitao $\mathrm{Lv}^{1,2^{*}}$
}

Heteroatom doping is an efficient way to tailor the electrochemical properties of carbon-based energy storage materials. However, synthesizing nitrogen-doped carbon materials with high doping level (e.g. over 10.0 at.\% $\mathrm{N}$ content) and controllable doping contents is very challenging. Herein, a tablet-sintering method using two nitrogen sources (ammonia and urea) is proposed to realize the regulation of nitrogen contents in carbon materials from 4.8 at.\% to almost 12.0 at.\%. Optimized sample with 10.1 at.\% nitrogen content demonstrates excellent pseudocapacitive behaviors with $104.1 \mathrm{~F} \mathrm{~g} \mathrm{~g}^{-1}$ and $176.3 \mathrm{~F} \mathrm{~g}^{-1}$ for the lithium and sodium-ion capacitors in organic electrolytes at $1 \mathrm{~A} \mathrm{~g}^{-1}$. Moreover, highly doped nitrogen could promote the cycling stability of carbon-based materials at high voltage (up to $5.0 \mathrm{~V}$ ) in the ionic liquid electrolyte. This may open a new avenue for the chemical doping of carbon-based materials and their applications in high-performance electrochemical devices.

Keywords: Chemical doping; Pseudocapacitance; Lithium/sodium-ion capacitors

Received 4 January 2019, Accepted 27 January 2019

DOI: $10.30919 / \mathrm{esee} 8 \mathrm{c} 212$

\section{Introduction}

Highly efficient electrochemical energy storage is crucial for the development of portable electronics, electric vehicles and utilizations of renewable energies (e.g. solar and wind energy). ${ }^{14}$ As one of the most important electrochemical energy storage devices, supercapacitors have the merits of high power density, long working life ( $>10$ years) and wide temperature range $\left(-20 \sim 70{ }^{\circ} \mathrm{C}\right) .^{5.7}$ However, the currently-used activated carbon-based supercapacitors with electrical double-layer capacitance (EDLC) mainly store energy by the surface absorption and deliver limited specific capacities. ${ }^{8,9}$ To cope with this issue, lithium/sodium-ion capacitors (LICs/NICs) have attracted increasing research attentions by combining the high power density of supercapacitors and high energy density of batteries. ${ }^{10-12}$ So far, different materials (e.g. MXenes, ${ }^{13,14} \mathrm{Nb}_{2} \mathrm{O}_{5}$, ${ }^{12,15}$ and some other two-dimensional (2D) materials ${ }^{16,17}$ ) have been explored for LICs/NICs applications. Compared with above-mentioned materials which are usually based on the ion intercalation mechanism, the carbon-based pseudocapacitive materials store energy by combining the surface absorption and surface redox reactions, which will lead to much better energy-power properties. However, in order to achieve better kinetic diffusions in the electrodes, a rational design of the carbon-based materials is very crucial. $^{18,19}$

As an important 2D-based carbon material, graphene has been

${ }^{1}$ State Key Laboratory of New Ceramics and Fine Processing, School of Materials Science and Engineering, Tsinghua University, Beijing 100084, China

${ }^{2}$ Key Laboratory of Advanced Materials (MOE), School of Materials Science and Engineering, Tsinghua University, Beijing 100084, China

*E-mail: lvruitao@tsinghua.edu.cn widely investigated for the applications in lithium-ion batteries (LIBs), ${ }^{20,21}$ supercapacitors, ${ }^{22,23}$ and electrocatalysts. ${ }^{2426}$ So far, various methods for the synthesis of graphene and its derivatives (e.g. graphene oxide (GO), including chemical vapor deposition (CVD), mechanical exfoliation, ${ }^{27}$ epitaxial growth ${ }^{28}$ and the modified Hummers method ${ }^{29}$ have been proposed. Among them, the modified Hummers method has been considered as the most promising one for the scale-up production of graphene and its derivatives for the energy storage device applications. However, the GO products synthesized from these methods usually possess high content of oxygen-containing functional groups (over $30 \mathrm{wt} \%$ ) on the surface, which is not ideal for their applications in EDLC. Kaner et $a l^{30}$ confirmed that the reduction of oxygen-containing functional groups on the surface of graphene oxide leads to a much enhanced electrochemical performance for the supercapacitor application. In addition, our recent work $^{31}$ has demonstrated that heteroatom (e.g. nitrogen) doping is an efficient way to improve the electrochemical properties of pristine GO. Nitrogen doping could not only improve the conductivity by decreasing the amount of oxygen and increasing the concentration of charge carriers, ${ }^{31}$ but also can optimize the contact between the electrode and electrolyte. ${ }^{32}$ Recent studies have confirmed that $\mathrm{N}$-doped carbon materials are promising candidates for the next-generation high-performance capacitors. ${ }^{26}$ The synergetic effect between doped nitrogen atoms and graphitic carbon will greatly promote the energy storage abilities. ${ }^{33-35}$ However, it is very challenging to achieve high doping level (e.g. $>10.0$ at. $\% \mathrm{~N}$ content) by traditional doping methods. In addition, it is also difficult to control the exact nitrogen doping contents by traditional gassolid phase reaction due to the random surface adsorption of the external gaseous dopant precursors onto the carbon matrix (solid).

In this work, we proposed a novel tablet-sintering method with urea (solid) and ammonia (gas) as "internal" and "external" nitrogen sources and obtained doped GO with controllable nitrogen contents. As- 
synthesized samples possess nitrogen contents from 4.8 at.\% to almost 12.0 at.\%. The optimized nitrogen doping leads to much enhanced electrochemical properties in organic, aqueous solution and ionic liquid electrolytes, which may open a new avenue for the research on the chemical doping of carbon-based materials and their electrochemical device applications.

\section{Experimental section}

\subsection{Sample synthesis}

GO powders were synthesized via a modified Hummers method. $10 \mathrm{~g}$ flake graphite powder and $5 \mathrm{~g} \mathrm{NaNO}_{3}$ were mixed in $230 \mathrm{~mL} \mathrm{H}_{2} \mathrm{SO}_{4}$ (98 wt. \%) at $0{ }^{\circ} \mathrm{C}$ in an ice-bath followed by the addition of $30 \mathrm{~g}$ $\mathrm{KMnO}_{4}$ under vigorous stirring at $0{ }^{\circ} \mathrm{C}$. The temperature of water bath was then changed to $\sim 25^{\circ} \mathrm{C}$ and maintained for $30 \mathrm{~min}$. The deionized water was slowly added into the above mixture and the temperature was kept at no more than $100{ }^{\circ} \mathrm{C}$. After this step, the mixture was stirred at $98{ }^{\circ} \mathrm{C}$ for $3 \mathrm{~h}$ followed by further addition of $30 \mathrm{~mL}$ of $30 \% \mathrm{H}_{2} \mathrm{O}_{2}$ aqueous solution. Finally, the products were obtained after subsequent centrifugation, washing and drying treatments. For the synthesis of graphene oxide (GO), the graphite oxide powders were treated under a sonication in a cell shredder and then collected for use.

The doped graphene oxides with controllable nitrogen contents were achieved by a bi-source doping strategy as illustrated in Scheme 1. GO and urea powders were mixed uniformly with different mass ratios and pressed into tablets. These tablets were then put in the middle of a tube furnace with argon atmosphere. When the temperature reached $600{ }^{\circ} \mathrm{C}$, the flow of Ar was switched to $\mathrm{NH}_{3}$ and kept for $60 \mathrm{~min}$. Assynthesized samples were then collected and rinsed with de-ionized water for several times. Finally, the samples were dried at $80{ }^{\circ} \mathrm{C}$ in a vacuum oven overnight. The samples prepared by this bi-source doping strategy were denoted as $\mathrm{G}_{x} \mathrm{U}_{y}$ where $x$ and $y$ are the mass ratios of $\mathrm{GO}$ and urea, respectively. For example, G1U0 means the mass ratio of GO and urea is 1:0. For comparison, direct ammonia treatment (i.e. singlesource doping) of graphene oxide powder was also carried out and denoted as NGO.

\subsection{Sample characterizations}

The crystalline structures of obtained samples were characterized by Xray diffraction (XRD, Rigaku, D/Max-2500/PC) measurement using $\mathrm{Cu}-\mathrm{K} \alpha$ radiation. Raman spectra (LabRAM,HR Evolution) were used to characterize the defects in carbon-based materials. The morphologies of as-synthesized samples were investigated with a field-emission scanning electron microscopy (FE-SEM, Hitachi S4800) and a transmission electron microscopy (TEM, JEOL 2010), both of which were equipped with energy dispersive spectrometers (EDS). The specific surface area (SSA) and pore structures were characterized by the nitrogen adsorption/desorption at $77 \mathrm{~K}$ (Belsorp-Max/Mini). The pore size distributions were calculated by the density functional theory (DFT) method. X-ray photoelectron spectroscopy (XPS) data were collected with an ESCLab 250X followed by calibrating the binding energy with C1s $(284.8 \mathrm{eV})$

\subsection{Electrochemical tests}

Electrochemical properties of as-obtained samples were tested in the stainless-steel 2032-coin cells. The electrodes with $\sim 1.2 \mathrm{mg} \mathrm{cm}^{-2}$ loading mass were prepared by coating the slurries containing the active materials (90 wt.\%) and polyvinylidene diflouride (PVDF) (10 wt.\%) onto the surface of aluminum foils and dried at $120{ }^{\circ} \mathrm{C}$ in vacuum overnight. Half-cells were assembled in an argon-filled glove box with metal $\mathrm{Na} / \mathrm{Li}$ foils as the counter electrodes. Electrolytes for LICs and NICs are $\mathrm{LiPF}_{6}(1 \mathrm{M})$ and $\mathrm{NaClO}_{4}(1 \mathrm{M})$ in the ethylene carbonate (EC) and dimethyl carbonate (DMC) (volume ratio of $\mathrm{EC}$ : $\mathrm{DMC}=1: 1$ ), respectively. The NICs were tested at the potential range of 1.5-4.2 V ( vs. $\mathrm{Na}^{+} / \mathrm{Na}$ ). Because of the lower standard potential of Li, LICs were tested at the potential ranging from $1.8 \mathrm{~V}$ to $4.5 \mathrm{~V}\left(v \mathrm{~s} . \mathrm{Li}^{+} / \mathrm{Li}\right)$ on Land battery testing systems. Meanwhile, the ionic liquid electrolyte was obtained by dissolving lithium bis(trifluoromethylsulphonyl)imide (LiTFSI) in 1-ethyl-3-methylimidazolium-bis(trifluoromethylsulfonyl)imide) (EMITFSI). It was used to investigate the electrochemical properties under high-voltage $(5 \mathrm{~V})$. The electrochemical properties of as-obtained

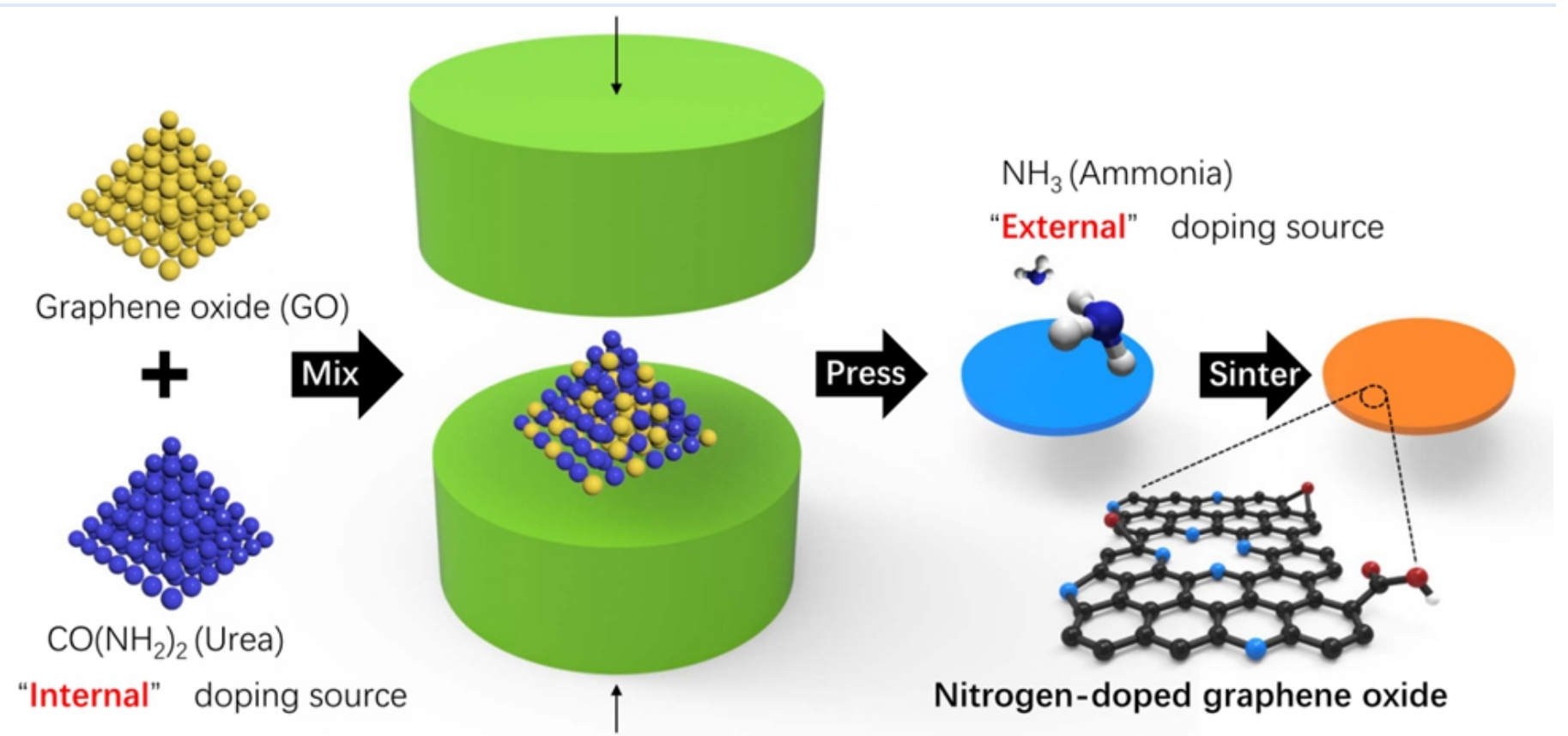

Scheme 1. Schematic illustration of a bi-source doping synthesis of nitrogen-doped graphene oxide. Graphene oxide (GO) and urea powders are mixed and pressed into a tablet, then sintered in $\mathrm{NH}_{3}$ atmosphere. Here urea powders (solid) and ammonia (gas) are used as the "internal" and "external" nitrogen sources for the controllable doping of GO (solid), respectively. 
samples in aqueous solution (6 $\mathrm{M} \mathrm{KOH}$ as electrolyte) were also investigated systematically. To elucidate the energy storage mechanism, the electrochemical impedance spectroscopy (EIS) with the frequency from $100 \mathrm{kHz}$ to $10 \mathrm{mHz}$ and cyclic voltammetry (CV) techniques at related voltage windows were conducted on an electrochemical workstation (EC-Lab, Bio-shop).

\section{Results and discussion}

The synthetic strategy of highly doped graphene oxide was inspired by the traditional fabrication process of advanced ceramics. To obtain a dense ceramic material, precursor powders are usually pressed into tablets followed by sintering. In our case, graphite oxide and urea powders were mixed uniformly and pressed into tablets and then sintered in $\mathrm{NH}_{3}$ atmosphere. The phase and structures of as-prepared samples were characterized as shown in Fig. 1. There exhibit three typical characteristic peaks at $1340 \mathrm{~cm}^{-1}, 1580 \mathrm{~cm}^{-1}$ and $2680 \mathrm{~cm}^{-1}$ in the Raman spectra, corresponding to the D-band (disorder-induced), G-band (in-plane vibration) and 2D band of carbon-based materials (Fig. 1(a)). ${ }^{36}$ The intensity ratio of $\mathrm{D}$ and $\mathrm{G}$ peak $\left(\mathrm{I}_{D} / \mathrm{I}_{G}\right)$ is used to index the degrees of graphitic ordering of carbon materials. As shown in Fig. 1(a), $\mathrm{I}_{D} / \mathrm{I}_{G}$ ratios are $1.21,1.15$ and 1.13 for G1U2, G1U0 and NGO, respectively. The higher $\mathrm{I}_{D} / \mathrm{I}_{G}$ value of G1U2 reveals the more disordered structure than G1U0 and NGO, which might be attributed to the high nitrogen doping level due to the contribution from urea as an "internal" doping source. XRD patterns of G1U2 along with G1U0 and NGO are shown in Fig. 1(b). The broad peaks at around $25^{\circ}$ and $43^{\circ}$ can be indexed to the crystallographic planes of (002) and (100) in the disordered carbon structures. ${ }^{37}$ With the assistance of the Scherrer equation, it could be calculated that the average values of interlayer spacings are $0.342 \mathrm{~nm}$, $0.354 \mathrm{~nm}$ and $0.346 \mathrm{~nm}$ for NGO, G1U0 and G1U2, respectively. By comparing the XRD patterns of different samples, it can be found that the (002) peaks will gradually broaden with the increase of urea contents (Fig. S1). Related data obtained from XRD analysis are listed in Table S1. FT-IR analysis confirms the introduction of nitrogencontaining groups in doped GO samples (Fig. 1 (c)). The peaks at around $3420 \mathrm{~cm}^{-1}$ could be indexed to the stretching vibrations of $\mathrm{O}-\mathrm{H}$ or $\mathrm{N}-\mathrm{H}^{31}$ As marked in Fig. 1(c), the following functional groups were confirmed in these three samples: $-\mathrm{CHO}$ or $-\mathrm{CH}$ stretching vibrations $\left(2700-3000 \mathrm{~cm}^{-1}\right)$; $-\mathrm{COOH} /-\mathrm{C}=\mathrm{N}$ - stretching vibrations $(1700-1740 \mathrm{~cm}$ $\left.{ }^{1}\right)$; $C=C$ stretching vibrations of $s p^{2}$ carbon $\left(1590-1650 \mathrm{~cm}^{-1}\right)$. XPS technique was carried out to analyze the nitrogen doping levels in different samples. XPS survey scan spectra shown in Fig. 1(d) reveal that there only exist elements of $\mathrm{C}, \mathrm{N}$ and $\mathrm{O}$ with the nitrogen doping levels of 10.1 at.\%, 7.4 at.\% and 4.8 at.\% in G1U2, G1U0 and NGO, respectively. Fig. 1(e) shows the nitrogen adsorption-desorption isotherms of NGO, G1U0 and G1U2. The relevant pore size distributions are shown in Fig. 1(f). The adsorption-desorption isotherms of all as-prepared samples display type-IV hysteresis loops,
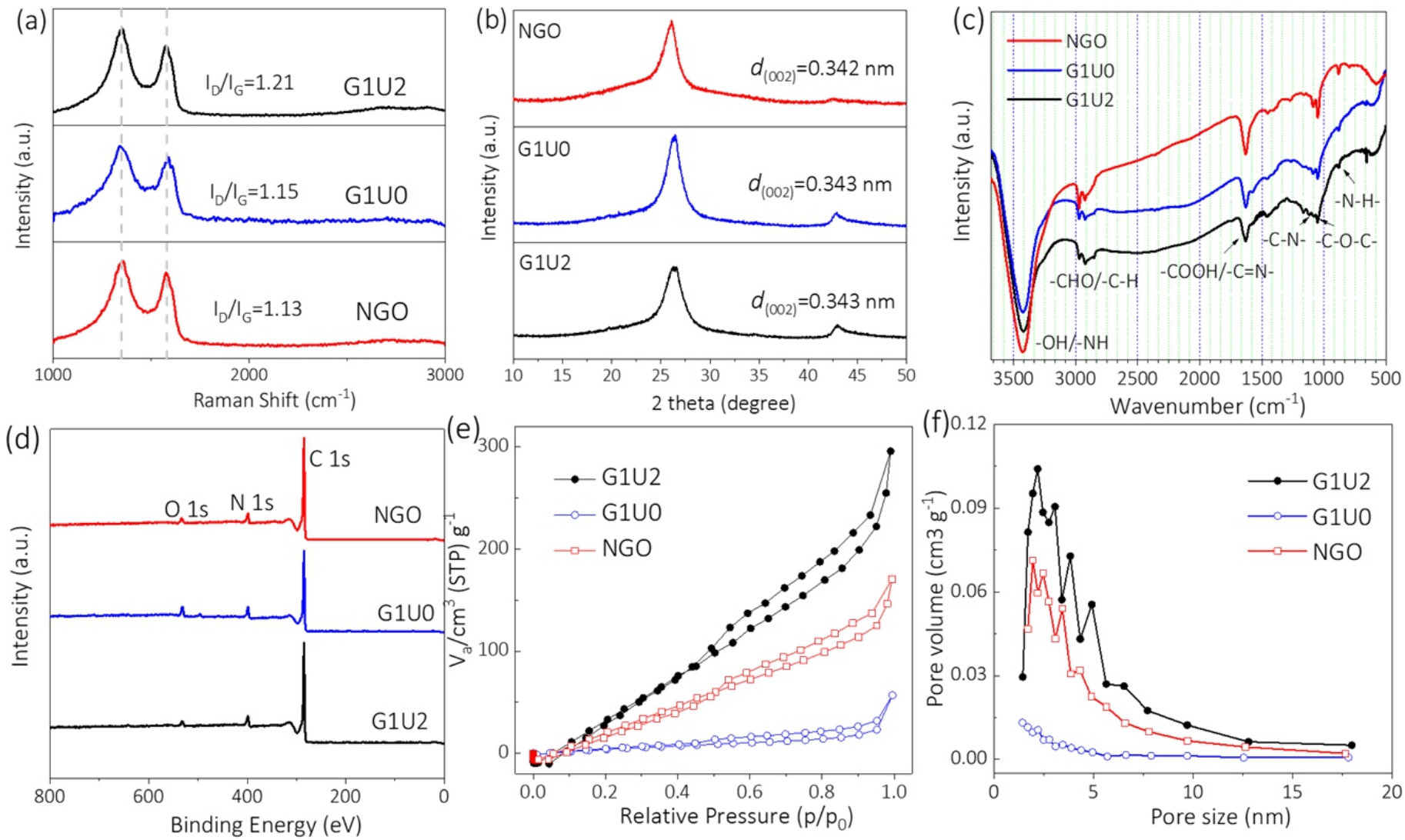

Fig. 1 Structural characterizations of different nitrogen-doped graphene oxide samples. (a) Raman spectra, (b) X-ray diffraction (XRD) patterns, (c) Fourier transform infrared (FT-IR) spectra, (d) X-ray photoelectron spectroscopy (XPS) survey scan spectra, (e) nitrogen adsorption-desorption isotherms, (f) pore size distributions of sample G1U2, G1U0 and NGO. Here NGO denotes nitrogen doped graphene oxide synthesized by the direct ammonia treatment (i.e. single-source doping) without being pressed into a tablet. The samples prepared by the tablet-sintering method are denoted as $\mathrm{G}_{x} \mathrm{U}_{y}$ where $x$ and $y$ are the mass ratios of $\mathrm{GO}$ and urea, respectively. For example, G1U0 denotes the mass ratio of graphene oxide and urea is 1:0; G1U2 denotes the mass ratio of graphene oxide and urea is 1:2. 
which are usually observed in materials with sheet-like structures and slit-shaped pores. ${ }^{31}$ Besides, the adsorption-desorption isotherms of G3U1, G1U1 and G1U3 were also tested and shown in Fig. S2 which reveals the same characteristics as Fig. 1 (e).

Table S2 summarizes the SSA, pore volumes and average pore sizes of as-obtained samples. The increase of urea contents will effectively increase the SSA and pore volume of samples, which would result in better electrochemical properties. Since NGO was not pressed into pellets, more enough interaction with $\mathrm{NH}_{3}$ makes it possess a higher SSA and larger pore volume than those of G1U0. The pores sized from $1 \mathrm{~nm}$ to $5 \mathrm{~nm}$ could be detected in both G1U2 and G1U0 as shown in Fig. 1 (f). However, this phenomenon is not that obvious in G1U0 sample. Gogotsi et al..$^{38}$ proposed that the micropores in carbon will provide additional double-layer capacitance. Benefiting from abundant micropores and the small average pore size, G1U2 is promising to exhibit excellent electrochemical properties. Morphologies of resulted nitrogen doped GO were characterized using TEM and SEM techniques which are shown in Figs. S3-S5. Typical layered structures with smooth surface can be seen in all these samples (Figs. S3 and S5). Higher resolution images in Figs. S3 (b, d, f) and Figs. S4 (b, d, g) show that the thicknesses of the stacked graphene layers are about 5-10 $\mathrm{nm}$. The detailed pore parameters of different samples are listed in Table S2. The high-resolution TEM image in Fig. S4 reveals that the lattice spacing of G1U2 is about $0.346 \mathrm{~nm}$, corresponding to the results obtained by the Scherer equation from XRD analysis. Selected area electron diffraction (SAED) pattern of G1U2 in Fig. S4 (h) shows typical polycrystalline diffraction rings of reduced graphene-oxide based materials.

The nitrogen doping levels of G1U2, G1U0 and NGO by ICP-MS analysis are 10.4 at.\%, 7.1 at.\% and 4.6 at.\% (Fig. 2 (a)), which are in agreement with the XPS analysis. As demonstrated in Fig. 2 (b), the nitrogen contents will gradually increase with the increased amounts of urea from 4.8 at.\% to almost 12.0 at.\%. Meanwhile, the surface oxygencontaining groups are also partly removed with the increased urea amounts as shown in Fig. 2 (b). By deconvoluting the C1s peaks (Figs. $3(\mathrm{a}-\mathrm{c}))$, the contents of $\mathrm{C}=\mathrm{C}$ species calculated based on the integral area ratios are $73.2 \%, 67.1 \%$ and $72.8 \%$ in G1U2, G1U0 and NGO, respectively. The increased contents of $\mathrm{C}=\mathrm{C}$ bonds will increase the conductivity of samples thus lead to better electrochemical properties. ${ }^{26,32}$ High-resolution N1s spectra of G1U2, G1U0 and NGO are shown in Figs. 3 (d-f). Different nitrogen species are illustrated in Fig. 2 (c), which are mainly the pyridinic nitrogen $(\mathrm{N}-6)$, pyrrolic nitrogen $(\mathrm{N}-5)$ and graphitic nitrogen (N-Q). Bandosz et $a l^{3}$ reported that the pyridinic nitrogen and pyrrolic nitrogen will contribute to an enhanced capacitance due to their positive charges compared with graphitic nitrogen. The integral calculations of peak areas confirm that the total contents of pyridinic nitrogen and pyrrolic nitrogen in G1U2, G1U0 and NGO are $93.75 \%, 84.52 \%$ and $77.62 \%$, respectively. The ratios of
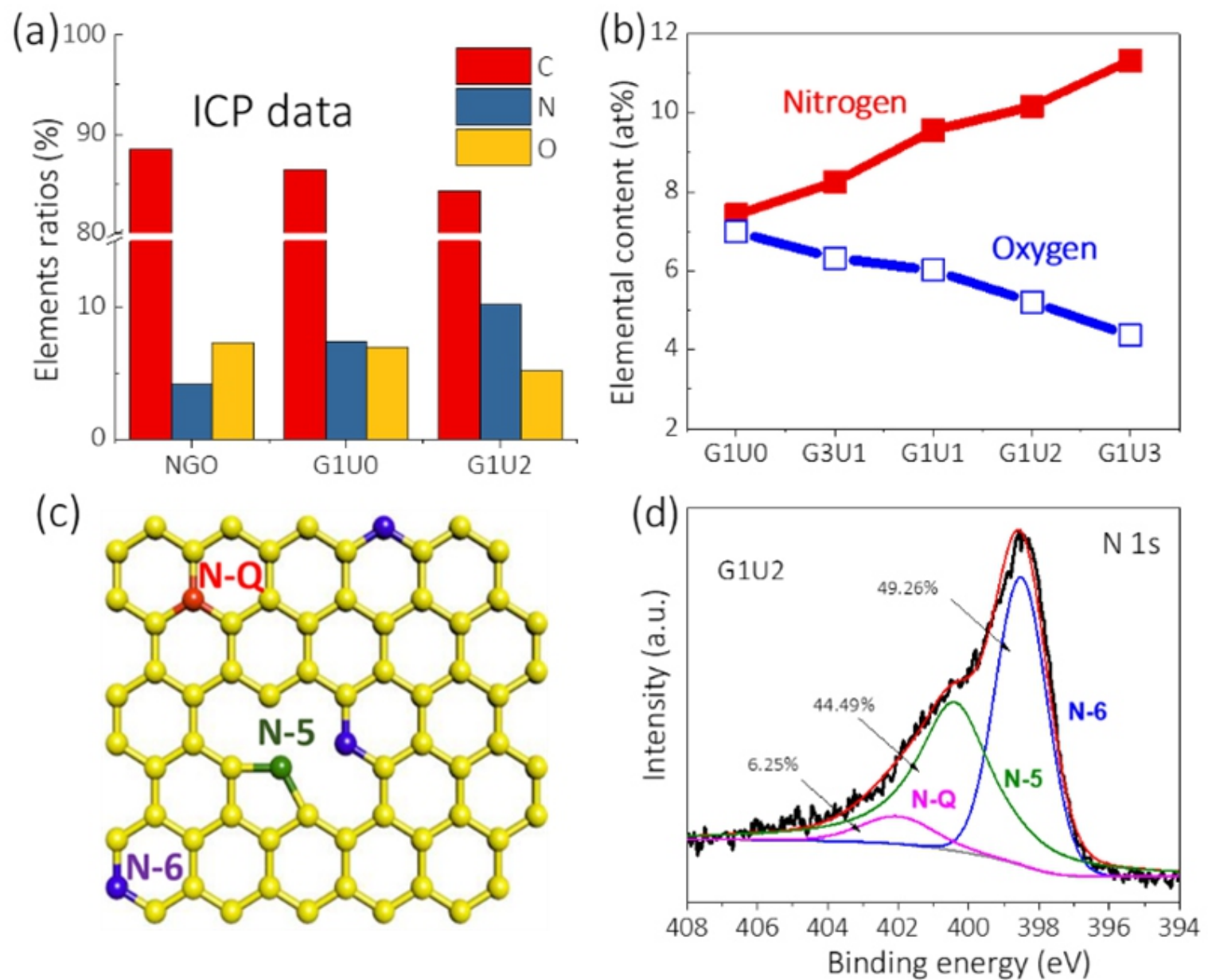

Fig. 2 (a) Inductively coupled plasma mass spectrometry (ICP-MS) analysis of C, N and O elements in NGO, G1U0 and G1U2 samples. (b) Nitrogen and oxygen elemental contents in different samples. (c) Schematic diagram of nitrogen doped graphene; Pyridinic N (N-6), pyrrolic N (N-5) and graphitic N (N-Q). (d) High-resolution XPS spectra of N 1s in the G1U2 samples. 

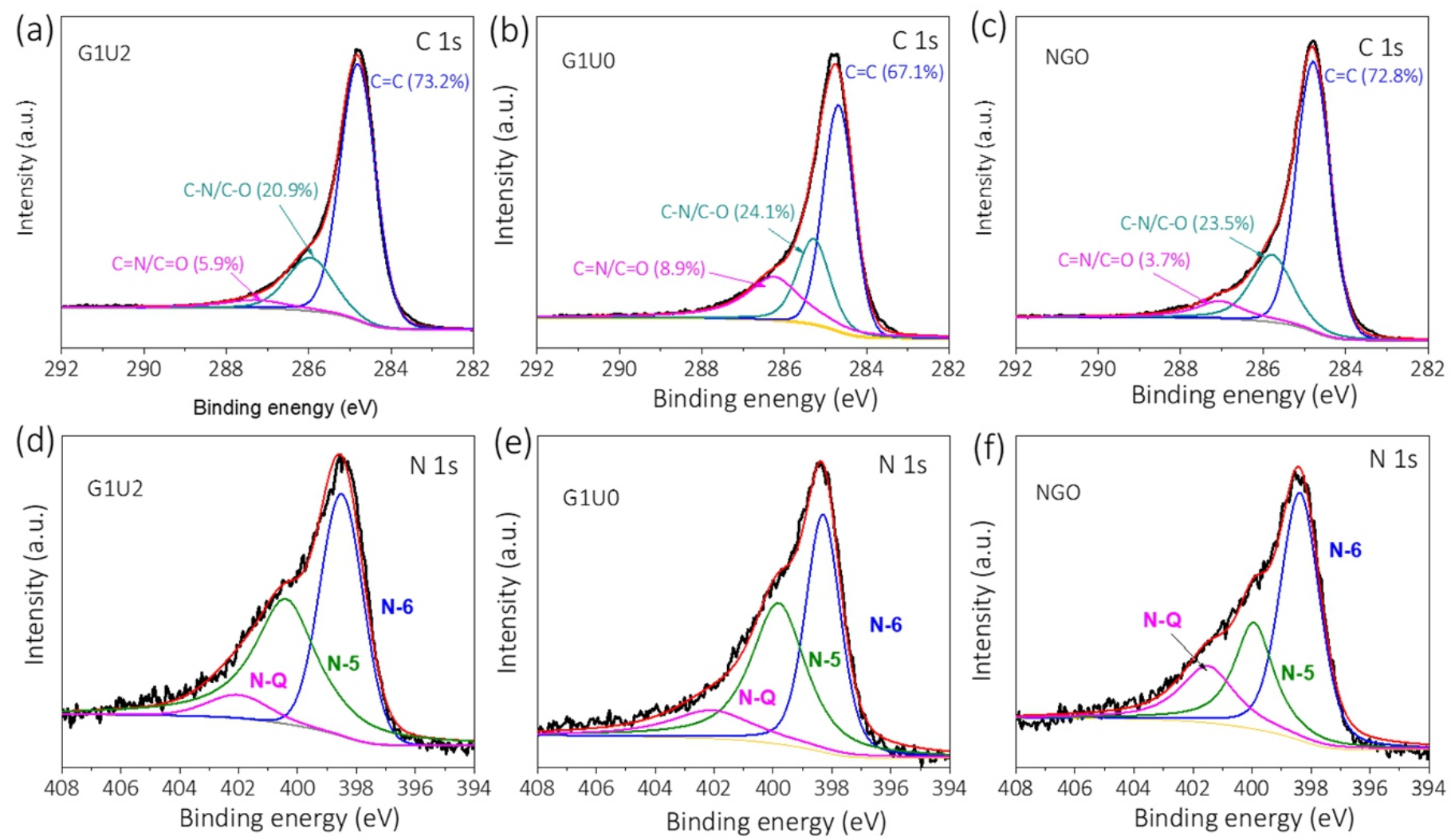

Fig. 3 High-resolution X-ray photoelectron spectroscopy (XPS) spectra of G1U2; G1U0 and NGO samples: (a-c) C1s; (d-f) N1s.

pyridinic nitrogen and pyrrolic nitrogen will increase with the increase of the amount of solid nitrogen source (urea), which will be important for the enhancement of the electrochemical performance of as-obtained samples. As shown in Fig. 2(d), the ratios of pyridinic nitrogen (N-6), pyrrolic nitrogen (N-5) and graphitic nitrogen (N-Q) in G1U2 are 6.25 $\%, 44.49 \%$ and $49.26 \%$, respectively. It is expected that the G1U2 could deliver a competitive performance as electrode in capacitors.

As-synthesized sample was furtherly investigated as energy storage materials in LICs/NICs and aqueous supercapacitors. Firstly, the electrochemical properties of all these samples are investigated as the cathodes of NICs in the potential range from $1.5 \mathrm{~V}$ to $4.2 \mathrm{~V}$ at $1 \mathrm{~A} \mathrm{~g}^{-1}$. As demonstrated in Fig. S6, the capacity of NGO is $42 \mathrm{mAh} \mathrm{g}^{-1}$, much higher than that (17 $\left.\mathrm{mAh} \mathrm{g}^{-1}\right)$ of G1U0. Tablet-sintering samples show that the capacities increase first and then decrease as shown in Fig. S6. As the cathode of NIC, the G1U2 sample demonstrates the best electrochemical capacity among all these doped GO samples with $\sim 82$ $\mathrm{mAh} \mathrm{g}^{-1}$. Galvanostatic charge-discharge (GCD) curves of G1U2, G1U0 and NGO as the cathodes of LICs and NICs in organic electrolyte systems are shown in Fig. 4. In Fig. 4(a), G1U2 could deliver about 104.1 $\mathrm{F} \mathrm{g}^{-1}$ specific capacitance at $1 \mathrm{~A} \mathrm{~g}^{-1}$ in the voltage window ranging from $1.8 \mathrm{~V}$ to $4.5 \mathrm{~V}$ in LICs. Meanwhile, both G1U0 and NGO only deliver $36.1 \mathrm{~F} \mathrm{~g} \mathrm{~g}^{-1}$ capacitances in LICs, indicating the enhanced electrochemical capacitances by high content of nitrogen doping. As shown in Fig. 4(b), the cyclic voltammetry (CV) curves of G1U2, G1U0 and NGO electrodes in LICs display rectangular shapes, which are the typical EDLC behaviors. Besides, several humps exist in the CV curves (Fig. 4(b)), demonstrating the surface redox reactions of pseudocapacitances. The Nyquist plots (Fig. 4(c)) of half-cell LICs with open circuit states exhibit $45^{\circ}$ slopes in the low frequency region, agreeing with the EDLC behaviors of carbon-based materials. The solution resistances $\left(R_{s}\right)$ of G1U2, G1U0 and NGO electrodes in LICs are $4.5 \Omega, 4.7 \Omega$ and $12.1 \Omega$, respectively, indicative of prior contacts between the electrolyte and electrodes. Different from the Ohmic contact resistances, the charge-transfer resistances $\left(R_{c t}\right)$ are usually the measurement of ion conductivity, whose values can be calculated by the diameters of the semicircles in Nyquist plots in high-frequency region. Clearly, $R_{c t}$ of G1U2 is about $10 \Omega$, much smaller than those of G1U0 $(102 \Omega)$ and NGO (53 $\Omega$ ). Performances of as-obtained samples were also tested in the NIC systems using the same methods and the results are shown in Fig. 4(d). The GCD measurements of G1U2, G1U0 and $\mathrm{NGO}$ are carried out in the potentials ranging from $1.5 \mathrm{~V}$ to $4.2 \mathrm{~V}$ which is different from LICs due to the higher standard hydrogen electrode (SHE) potential of Li. It can becalculated that the capacitances of G1U2, G1U0 and NGO are $176.3 \mathrm{~F} \mathrm{~g} \mathrm{~g}^{-1}, 27.9 \mathrm{~F} \mathrm{~g}^{-1}$ and $77.8 \mathrm{~F} \mathrm{~g}^{-1}$, respectively. The capacitance of G1U2 is competitive compared with some recent works of carbon-based materials in capacitors. ${ }^{40-43}$

Different from the performance in LICs, the electrochemical performance of NGO is better than that of G1U0 in NICs, which may come from the inadequate contact between the surface of GO powders and ammonia molecules after a compaction process of G1U0. Moreover, this phenomenon could be explained from the EIS results in Fig. 4(f), which reveals the same trends as the Nyquist plots of LICs (Fig. 4(c)). Correspondingly, CV curves of G1U2, G1U0 and NGO in NICs shown in Fig. 4(e) display the same characteristics as the CV curves of LICs. The CV curve with rectangle shape indicates the existence of EDLCs while the humps overlaid the curves are from the pseudocapacitive reactions. Comparing the G1U2 electrochemical results of NICs and LICs in Fig. S7, it is found that the capacitance of NIC is about $60 \%$ larger than that of LIC. Because of the higher standard hydrogen electrode (SHE) potential $\left(\mathrm{Li}^{+} / \mathrm{Li}:-3.0 \mathrm{~V} ; \mathrm{Na}^{+} / \mathrm{Na}\right.$ : $2.7 \mathrm{~V}$ ) of $\mathrm{Li}$, the voltage potentials of LICs and NICs are set to be the same based on $\mathrm{Li}^{+} / \mathrm{Li}$ as shown in Figs. S7 (a, b). As EIS plots shown in 

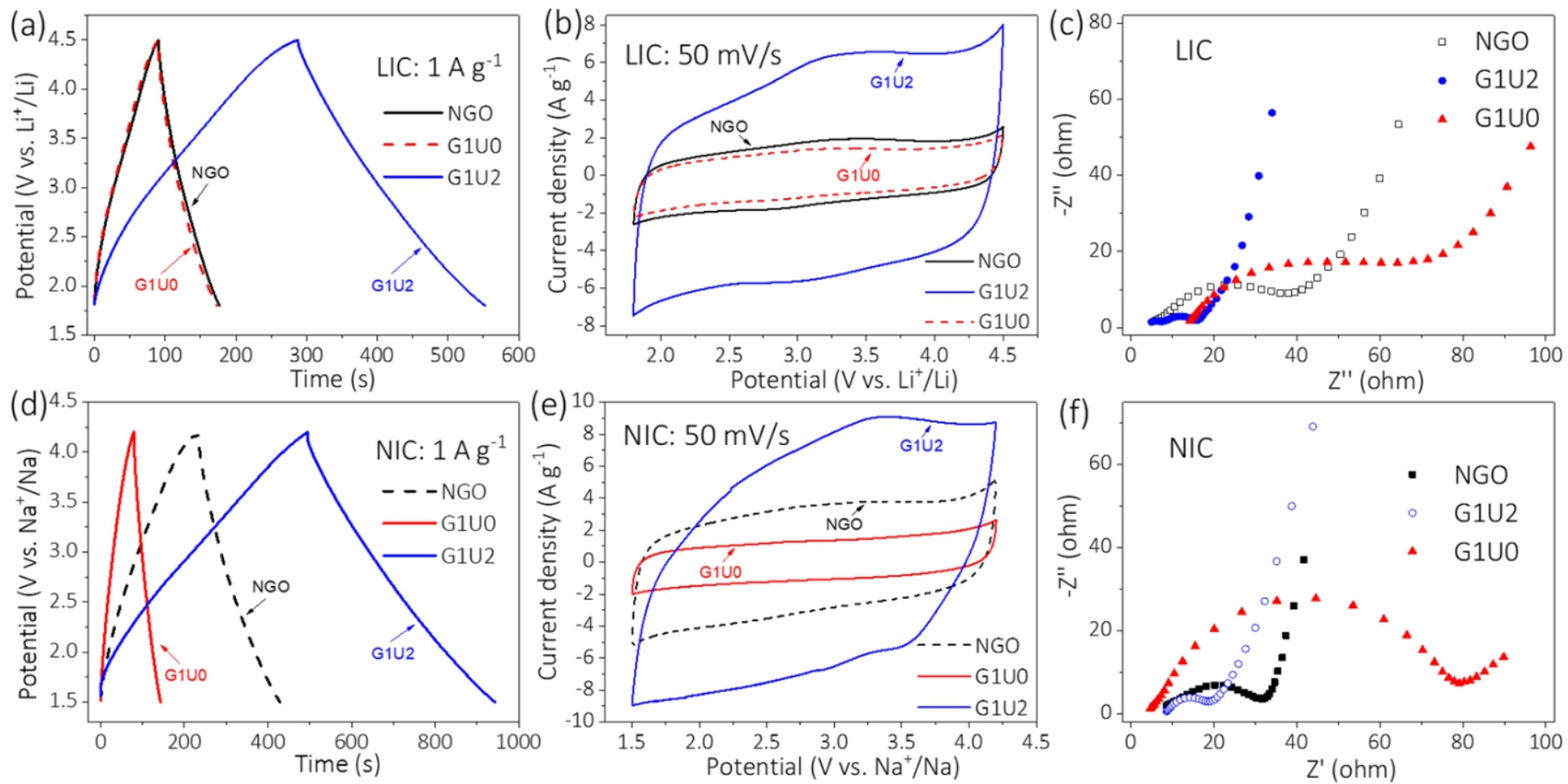

Fig. 4 Electrochemical properties of G1U2, G1U0 and NGO samples. (a) Comparison of Galvanostatic charge-discharge (GCD) curves as the cathodes of lithium ion capacitors (LICs) at $1 \mathrm{~A} \mathrm{~g}^{-1}$; (b) CV curves in LICs at as can rate of $50 \mathrm{mV} / \mathrm{s}$; (c) Comparison of EIS curves of as-obtained samples; (d) Comparison of GCD curves as the cathodes of sodium ion capacitors (NICs) at $1 \mathrm{~A} \mathrm{~g}^{-1}$; (e) Cyclic voltammetry (CV) curves in NICs at the scan rate of $50 \mathrm{mV} / \mathrm{s}$; (f) Comparison of Electrochemical impedance spectroscopy (EIS) curves of as-obtained samples in NICs.

Fig. S7(c), the charge transfer resistances of G1U2 in NIC and LIC are about 10, no obvious differences in both devices. After 3500 cycles and 2000 cycles in LICs and NICs, G1U2 could still deliver $\sim 60 \mathrm{mAh} \mathrm{g}^{-1}$ and $\sim 80 \mathrm{mAh} \mathrm{g}^{-1}$ capacities under $1 \mathrm{~A} \mathrm{~g}^{-1}$, respectively (Fig. S8). No obvious morphology changes can be found in the sample before and after the LIC and NIC tests, as shown in Fig. S9.

To investigate the capacity retention and the time constants of G1U2 in both LIC and NIC, the capacitors were firstly charged under 1 $\mathrm{A} \mathrm{g}^{-1}$ and then discharged under various current densities as exhibited in Fig. 5. The time constant $\left(\tau_{0}\right)$ was calculated using based on the following formula:

$$
\begin{aligned}
\tau_{0} & =R \cdot C \\
R & =\frac{U}{I}
\end{aligned}
$$

where $R$ is the resistance of electrode, $C$ is the capacitance, $U$ is the voltage drop, and $I$ is the current density. As demonstrated in Figs. 5(a) and (c), it can be calculated that the time constants of LIC and NIC are about $4.16 \mathrm{~s}$ and $10.58 \mathrm{~s}$, respectively. In a stable charge and discharge process, the charge and discharge capacities are mutually influenced by each other. High current discharge will cause a decrease in charge capacity at low currents as demonstrated in Figs. 5(b) and (d). The rate capacitances of G1U2 working as cathode for NIC are compared with some state-of-the-art carbon-based NIC electrodes as exhibited in Fig. $5(\mathrm{e}){ }^{42,4447}$ It can be seen that G1U2 could not only deliver high capacitance in low current densities, but also possess high capacity retention under high current densities.

The energy density $(E)$ of the hybrid device can be evaluated based on the formula as below:

$$
E=\frac{U \cdot I \cdot t}{m}
$$

where $U$ represents the working potential, $I$ represents the working current, and $m$ refers to the mass of active materials. Obviously, $E$ is proportional to the working potential $(U)$, indicating that the increase of $U$ would sharply enhance the electrochemical properties of the obtained devices. Herein, optimized samples G1U2 were investigated in the ionic liquid in the voltage ranging from $2.5 \mathrm{~V}$ to $5.0 \mathrm{~V}$ as shown in Fig. 6. When the potential reaches $5.0 \mathrm{~V}$, the anions intercalation would take place. When working at $1 \mathrm{~A} \mathrm{~g}^{-1}$, the Coulombic efficiency of G1U2 in ionic liquid is only about $87 \%$, which is mainly caused by the low Coulombic efficiency of anions intercalation (11.8 \%) as marked in Fig. 6(a). Beltrop et al. ${ }^{48}$ proposed that the size of anions and the cut-off potential will obviously influence the Coulombic efficiency of batteries. Moreover, both the electrochemical exfoliation and poor wetting ability between electrolyte and electrode will lead to the ultralow Coulombic efficiency in this system. ${ }^{49}, 50$ G1U2 could deliver $\sim 134.7 \mathrm{~F} \mathrm{~g}^{-1}, 78.9 \mathrm{~F} \mathrm{~g}^{-1}$ and $47.8 \mathrm{~F} \mathrm{~g}^{-1}$ capacities at $1 \mathrm{~A} \mathrm{~g}^{-1}, 2 \mathrm{~A} \mathrm{~g}^{-1}$ and $4 \mathrm{~A} \mathrm{~g}^{-1}$ as demonstrated in Figs. 6(b) and (c), indicating the poor rate performance in ionic liquid. Meanwhile, the kinetics of G1U2 in ionic liquid were investigated using CV method under various scan rates as shown in Fig. 6(d). Obviously, the areas of $\mathrm{CV}$ curves are proportional to the scan rates as shown in Fig. 6(d). The peak currents in CV curves obey a power-law relationship with the scan rates as follows:

$$
i=a v^{b}
$$

where $i$ is the peak current, $v$ is the scan rate, and $b$ values could be used to evaluate the reaction process of electrode. When $b=0.5$, it refers to a typical Faradaic intercalation process controlled by semi-infinite linear diffusion; when $b=1$, it represents a capacitive charge storage without diffusion-control mechanism. ${ }^{11,51}$ As shown in Fig. 6(e), when working at $3.5 \mathrm{~V}$ in ionic liquid, the $b$ values of G1U2 in oxidization and reduction processes are 0.74 and 0.68 , revealing the pseudocapacitive 

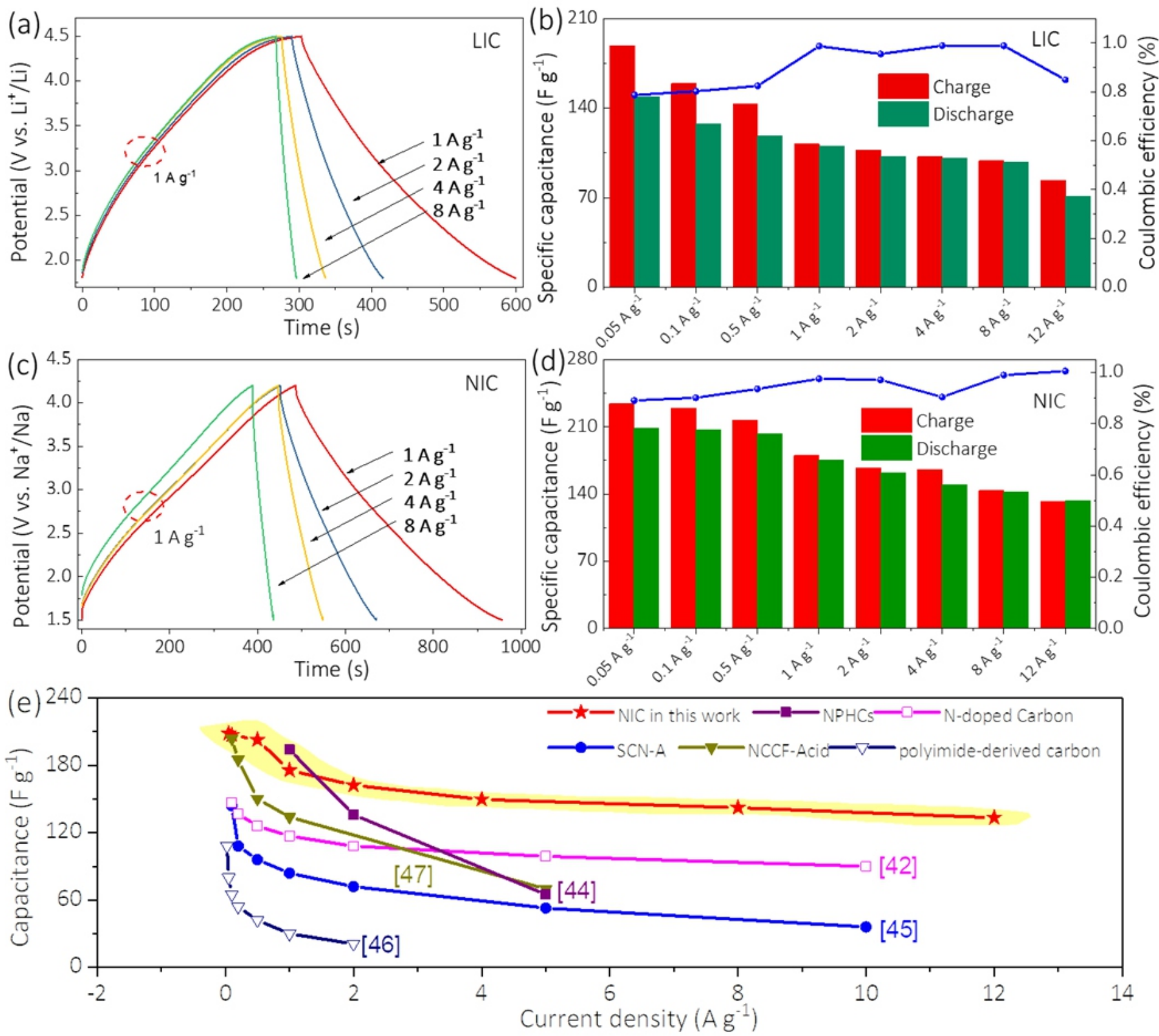

Fig. 5 (a), (c) GCD profiles of G1U2 in LIC and NIC with $1 \mathrm{~A} \mathrm{~g} \mathrm{~g}^{-1}$ charge current densities and different discharge current densities; (b), (d) Summary of charge and discharge capacities of samples at various current densities and their related Coulombic efficiencies. (e) Comparison of rate capacitance of G1U2 in NIC with other carbon-based materials. NPHCS: nitrogen-doped microporous hard carbons; ${ }^{44}$ SCN-A: peanut skin derived carbon nanosheets; ${ }^{45}$ NCCF-Acid: Nano cellular carbon foams tested in acid. ${ }^{47}$

behaviors of G1U2 sample. The electrochemical properties of G1U2 in ionic liquid were furtherly investigated by EIS analysis as shown in Fig. 6(f). Nyquist plots of G1U2 demonstrate that the solution resistance $\left(R_{s}\right)$ in this system is about $21 \Omega$, much bigger than that in LIC (4.5 $\Omega$ ) and NIC $(9.0 \Omega)$ systems. In the inset of Fig.6(f), the semicircles in high frequency region display a flat shape which are caused by the rough surface of highly nitrogen-doped GO, consistent with the pseudocapacitance introduced surface reaction process. ${ }^{24,52}$

Fig. 7 reveals the electrochemical properties of G1U2 in aqueous solution $(6 \mathrm{M} \mathrm{KOH})$ between $-1.0 \mathrm{~V}$ and $0 \mathrm{~V}$ vs. $\mathrm{Hg} / \mathrm{HgO}$. $\mathrm{CV}$ curves under different scan rates (Fig. 7(a)) display typical EDLC behaviors with rectangular shapes. G1U2 could deliver about 303, 285, 280, 254 and $234 \mathrm{~F} \mathrm{~g}^{-1}$ capacitances at 1, 2, 4, 20 and $40 \mathrm{~A} \mathrm{~g}^{-1}$ current densities.
Excellent cycling property of G1U2 can be also obtained as shown in Fig. 7(c). Compared with the initial GCD curve, there is only $12.5 \%$ decrease in capacitance after 3000 cycles in aqueous solution at a high current density of $4 \mathrm{~A} \mathrm{~g} \mathrm{~g}^{-1}$. The capacitance under specific potential can be calculated from the slopes of the lines between the current density and the scan rates based on the CV curves in Fig. 7(a). As shown in Fig. 7(d), the capacitances at different potentials are almost the same at about $300 \mathrm{~F} \mathrm{~g}^{-1}$, corresponding with the results of GCD curves. Benefiting from the ultralow resistance in aqueous solution (Fig. S10), G1U2 exhibits excellent rate performances with the increased scan rates as shown in Fig. 7(e). Even under the scan rate of $400 \mathrm{mV} / \mathrm{s}$, it could still deliver over $240 \mathrm{~F} \mathrm{~g}^{-1}$ capacitance. As shown in Fig. 7(f), compared with some recent works on carbon-based materials as electrodes for 

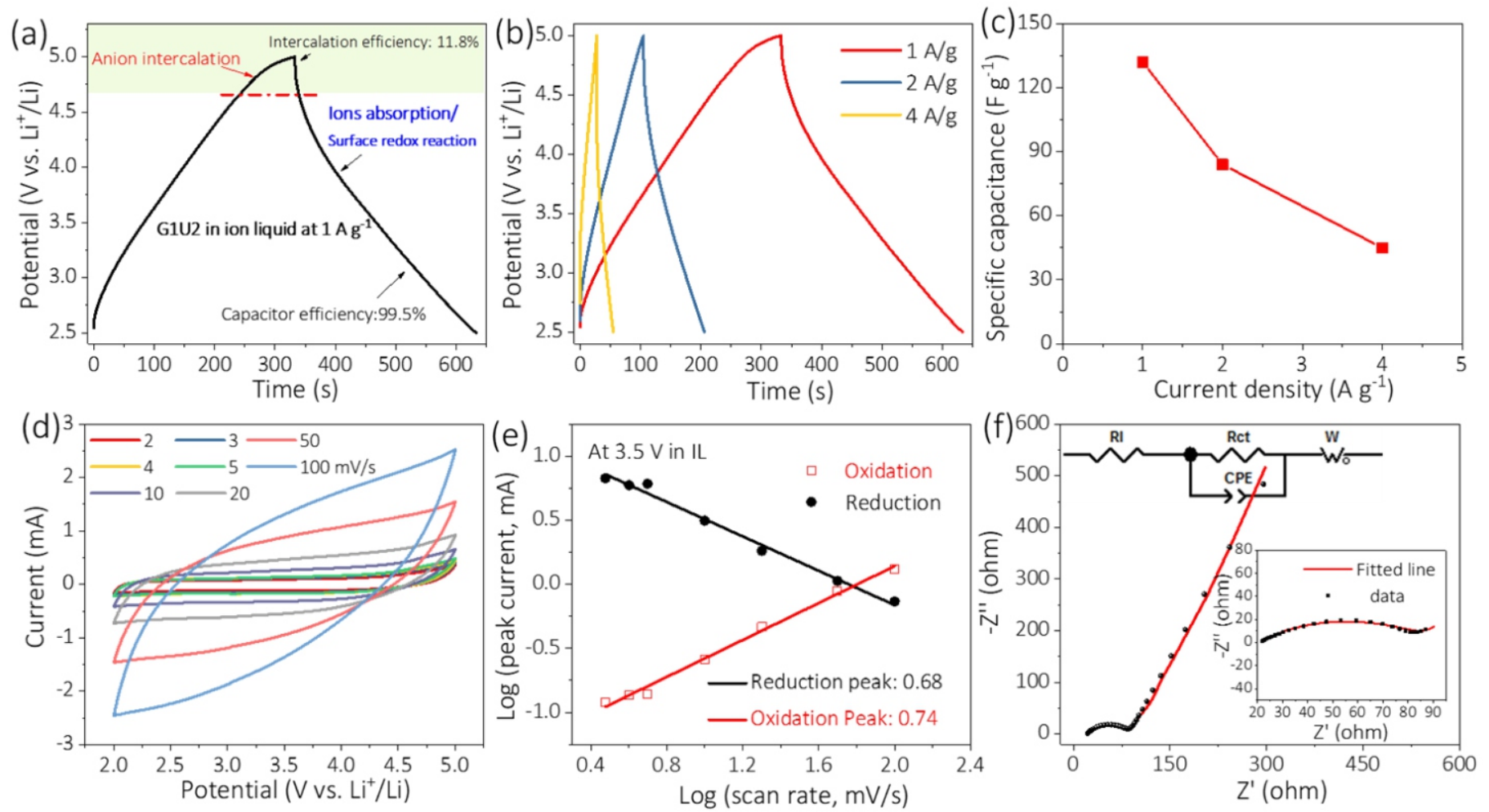

Fig. 6 (a) GCD curve of G1U2 in lithium ionic liquid at $1 \mathrm{~A} \mathrm{~g}^{-1}$ with the working voltages between $2.5 \mathrm{~V}$ and $5.0 \mathrm{~V}$; (b) GCD curves of G1U2 in lithium ionic liquid at 1, 2 and $4 \mathrm{~A} \mathrm{~g}^{-1}$; (c) Rate capacities of G1U2; (d) CV curves of G1U2 at various scan rates; (e) Determination of the $b$ values for various at $3.5 \mathrm{~V}$ for G1U2 in the ionic liquid electrolyte; (f) EIS data of G1U2 in ionic liquid and its fitted curve.
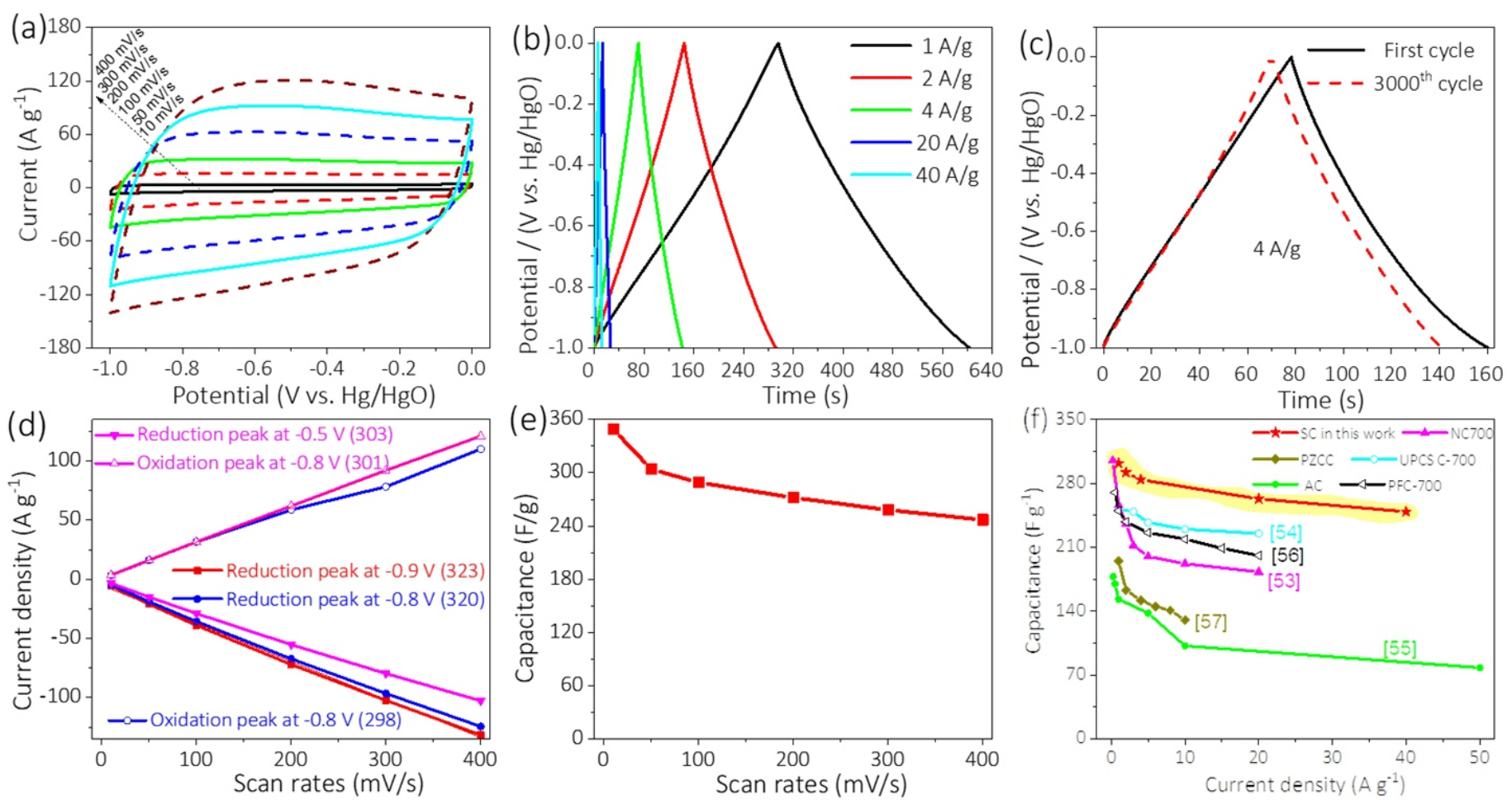

Fig. 7 (a) CV curves of G1U2 in aqueous solution under various scan rates; (b) Galvanostatic discharge/charge (GCD) profiles of G1U2 in aqueous solution at current densities from 1 to $40 \mathrm{~A} \mathrm{~g}^{-1}$; (c) GCD curves of G1U2 in the first and $3000^{\text {th }}$ cycles under $4 \mathrm{~A} \mathrm{~g}^{-1}$ in aqueous solution; (d) The sweep rate dependence on currents of G1U2 to characterize charge storage mechanisms. (e) Rate capacitance of G1U2 at different scan rates in aqueous electrolyte; (f) Comparison of rate capacitance of G1U2 in supercapacitor (SC) with other carbon-based materials. NC700: Cross-linked, N-doped, pillared-layered porous carbons obtained at $700{ }^{\circ} \mathrm{C} ;{ }^{53} \mathrm{PZCC}$ : $\mathrm{NaH}_{2} \mathrm{PO}_{4}-\mathrm{ZnCl}_{2}$-treated cellulose-derived carbon; $;$ PFC-700: Perilla frutescenleaf-derived carbon nanosheets; ${ }^{56} \mathrm{AC}$ : activated carbon; $;^{55}$ UPCS C-700: Ultrathinporous carbon shell synthesized by carbonization at $700{ }^{\circ} \mathrm{C}^{54}$ 
$\mathrm{SCs},{ }^{53-57}$ although some materials exhibit comparable performance to that of G1U2 at low current densities (e.g. 1, $2 \mathrm{~A} \mathrm{~g}^{-1}$ ), as-synthesized G1U2 demonstrates superior capacitance than the others at high current densities (e.g. 20, $40 \mathrm{~A} \mathrm{~g} \mathrm{~g}^{-1}$ ), which indicates their excellent rate performance.

\section{Conclusions}

In summary, we proposed a facile method to regulate the doping contents of nitrogen in carbon-based materials by a tablet-sintering route. The highly nitrogen-doped graphene oxides demonstrate excellent electrochemical properties for LICs/NICs in the ionic liquid and aqueous solution electrolytes. The optimized sample exhibits excellent pseudocapacitances of 104.1 and $176.3 \mathrm{~F} \mathrm{~g}^{-1}$ in the LICs and NICs at 1 $\mathrm{A} \mathrm{g}^{-1}$ with time constants of $10.58 \mathrm{~s}$ for NICs and $4.16 \mathrm{~s}$ for LICs, indicative of more remarkable pseudocapacitive behaviors in $\mathrm{Na}^{+}$ system. Moreover, the highly doped graphene oxide could be charged up to $5 \mathrm{~V}$, demonstrating superior anti-oxidation ability by introducing nitrogen-containing groups. The unique characteristics of highly doped graphene oxide in different types of capacitors reveal the potentials as candidates for the next-generation advanced energy storage devices.

\section{Conflict of interest}

There are no conflicts to declare.

\section{Acknowledgements}

We acknowledge the financial support from the National Basic Research Program of China (Grant No. 2015CB932500), National Natural Science Foundation of China (Grant No. 51722207) and the Tsinghua University Initiative Scientific Research Program (Grant No. 20151080367).We also thank Xiaona Guan and Hongwei Zhang for the fruitful discussion duringthe manuscript preparation.

\section{Reference}

1. P. Simon and Y. Gogotsi, Nat. Mater, 2008, 7, 845-854.

2. J. Y. Hwang, S. T. Myung and Y. K. Sun, Chem. Soc. Rev., 2017, 46, 35293614

3. C.Yan, Y. Zhu, Z. Fang, C. Lv, X. Zhou, G. Chen and G. Yu, Adv. Energy Mater., 2018,8, 1800762.

4. L. Peng, Z. Fang, Y. Zhu, C. Yan and G. Yu, Adv. Energy Mater., 2018, 8, 1702179

5. J. Y. Hwang, M. Li, M. F. El-Kady and R. B. Kaner, Adv. Funct. Mater, 2017, 27, 1605745

6. J. Xu, Y. Wang, S. Cao, J. Zhang, G. Zhang, H. Xue, Q. Xuand H. Pang. J. Mater. Chem. A, 2018, 6, 17329-17336.

7. B. Li, F. Dai, Q. Xiao, L. Yang, J. Shen, C. Zhang and M. Cai, Adv. Energy Mater, 2016, 6, 1600802.

8. S. Cao, B. Li, R. Zhu and H. Pang, Chem. Eng. J., 2019, 355, 602-623.

9. P. Geng, S. Zheng, H. Tang, R. Zhu, L. Zhang, S. Cao, H. Xue and H. Pang, Adv. Energy Mater, 2018, 8, 1703259.

10. P. Simon, Y. Gogotsi and B. Dunn, Science, 2014, 343, 1210-1211.

11. Z. Le, F. Liu, P. Nie, X. Li, X. Liu, Z. Bian, G. Chen, H. B. Wu and Y. Lu, ACS Nano, 2017, 11, 2952-2960.

12. J. W. Kim, V. Augustyn and B. Dunn, Adv. Energy Mater, 2012, 2, 141-148.

13. B. Anasori, M. R. Lukatskaya and Y. Gogotsi, Nat. Rev. Mater, 2017, 2, 16098 .

14. X. Wang, S. Kajiyama, H. Iinuma, E. Hosono, S. Oro, I. Moriguchi, M. Okubo and A. Yamada, Nat. Commun., 2015, 6, 6544.

15. X. Wang, G. Li, Z. Chen, V. Augustyn, X. Ma, G. Wang, B. Dunn and Y. Lu, Adv. Energy Mater., 2011, 1, 1089-1093.

16. Y. Luo, Y. Tang, S. Zheng, Y. Yan, H. Xue and H. Pang, J. Mater. Chem. A, 2018, 6, 4236-4259.

17. Y. Zhu, L. Peng, Z. Fang, C. Yan, X. Zhang and G. Yu, Adv. Mater, 2018,
30, 1706347.

18. H. Liu, H. Song, X. Chen, S. Zhang, J. Zhou and Z. Ma, J. Power Sources, 2015, 285, 303-309.

19. V. Augustyn, P. Simon and B. Dunn, Energy Environ. Sci., 2014, 7, $1597-$ 1614.

20. H. Gao, T. Zhou, Y. Zheng, Y. Liu, J. Chen, H. Liu and Z. Guo, Adv. Energy Mater., 2016, 6, 1601037.

21. J. Chang, X. Huang, G. Zhou, S. Cui, P. B. Hallac, J. Jiang, P. T. Hurley and J. Chen, Adv. Mater, 2014, 26, 758-764.

22. M. A. Bissett, I. A. Kinloch and R. A. W. Dryfe, ACS Appl. Mater. Inter, 2015, 7, 17388-17398

23. J. Yan, Z. Fan, W. Sun, G. Ning, T. Wei, Q. Zhang, R. Zhang, L. Zhi and F. Wei, Adv. Funct. Mater., 2012, 22, 2632-2641.

24. Z. Yang, Z. Yao, G. Li, G. Fang, H. Nie, Z. Liu, X. Zhou, X. a. Chen and S Huang, ACS Nano, 2012, 6, 205-211.

25. Z. Ma, S. Dou, A. Shen, L. Tao, L. Dai and S. Wang, Angew. Chem. Int. Ed., 2015, 54, 1888-1892.

26. F. Bonaccorso, L. Colombo, G. Yu, M. Stoller, V. Tozzini, A. C. Ferrari, R. S. Ruoff and V. Pellegrini, Science, 2015, 347.

27. M. Yi and Z. Shen, J. Mater. Chem. A, 2015, 3, 11700-11715.

28. W. Yang, G. Chen, Z. Shi, C. C. Liu, L. Zhang, G. Xie, M. Cheng, D. Wang, R. Yang, D. Shi, K. Watanabe, T. Taniguchi, Y. Yao, Y. Zhang and G. Zhang, Nat. Mater, 2013, 12, 792.

29. J. Chen, B. Yao, C. Li and G. Shi, Carbon, 2013, 64, 225-229.

30. M. F. El-Kady, V. Strong, S. Dubin and R. B. Kaner, Science, 2012, 335, 1326-1330.

31. M. Hu, H. Zhou, X. Gan, L. Yang, Z. H. Huang, D. W. Wang, F. Kang and R. Lv, J. Mater. Chem. A, 2018, 6, 1582-1589.

32. T. Lin, I.-W. Chen, F. Liu, C. Yang, H. Bi, F. Xu and F. Huang, Science, $2015,350,1508-1513$.

33. H. A. Cha, H. M. Jeong and J. K. Kang, J. Mater. Chem. A, 2014, 2, 5182 5186.

34. H.G. Wang, Z. Wu, F. L. Meng, D. L. Ma, X. L. Huang, L. M. Wang and X. B. Zhang, Chemsuschem, 2013, 6, 56-60.

35. J. Liang, Y. Jiao, M. Jaroniec and S. Z. Qiao, Angew. Chem. Int. Ed., 2012 51, 11496-11500.

36. J. Ding, H. Wang, Z. Li, A. Kohandehghan, K. Cui, Z. Xu, B. Zahiri, X. Tan, E. M. Lotfabad, B. C. Olsen and D. Mitlin, ACS Nano, 2013, 7, 11004 11015

37. Y. Li, Y. S. Hu, M. M. Titirici, L. Chen and X. Huang, Adv. Energy Mater, 2016, 6, 1600659-n/a

38. J. Chmiola, G. Yushin, Y. Gogotsi, C. Portet, P. Simon and P. L. Taberna Science, 2006, 313, 1760-1763.

39. D. Hulicova-Jurcakova, M. Seredych, G. Q. Lu and T. J. Bandosz, Adv. Funct. Mater, 2009, 19, 438-447.

40. H. Wang, C. Zhu, D. Chao, Q. Yan and H. J. Fan, Adv. Mater., 2017, 29, 1702093-n/a.

41. S. R. Sivakkumar and A. G. Pandolfo, Electrochim. Acta, 2012, 65, 280-287.

42. H. Wang, Y. Zhang, H. Ang, Y. Zhang, H. T. Tan, Y. Zhang, Y. Guo, J. B. Franklin, X. L. Wu, M. Srinivasan, H. J. Fan and Q. Yan, Adv. Funct. Mater, 2016, 26, 3082-3093.

43. W. J. Cao and J. P. Zheng, J. Power Sources, 2012, 213, 180-185

44. S. Chen, J. Wang, L. Fan, R. Ma, E. Zhang, Q. Liu and B. Lu, Adv. Energy Mater, 2018, 8, 1800140.

45. H. Wang, D. Mitlin, J. Ding, Z. Li and K. Cui, J. Mater. Chem. A, 2016, 4, 5149-5158.

46. Q. Zhao, D. Yang, A. K. Whittaker and X. S. Zhao, J. Power Sources, 2018, 396, 12-18.

47. Y. Shao, J. Xiao, W. Wang, M. Engelhard, X. Chen, Z. Nie, M. Gu, L. V. Saraf, G. Exarhos, J.-G. Zhang and J. Liu, Nano Lett., 2013, 13, 3909-3914.

48. K. Beltrop, P. Meister, S. Klein, A. Heckmann, M. Grünebaum, H.-D Wiemhöfer, M. Winter and T. Placke, Electrochim. Acta, 2016, 209, 44-55.

49. T. Placke, P. Bieker, F. Lux Simon, O. Fromm, H.-W. Meyer, S. Passerini and M. Winter, Z. Phys. Chem., 2012, 226, 391-407

50. J. Huesker, L. Froböse, A. Kwade, M. Winter and T. Placke, Electrochim. Acta, 2017, 257, 423-435.

51. B. Qu, C. Ma, G. Ji, C. Xu, J. Xu, Y. S. Meng, T. Wang and J. Y. Lee, $A d v$ Mater., 2014, 26, 3854-3859. 
52. X. Wang, G. Sun, P. Routh, D. H. Kim, W. Huang and P. Chen, Chem. Soc. Rev., 2014, 43, 7067-7098.

53. W. Tian, H. Zhang, H. Sun, M. O. Tadé and S. Wang, Carbon, 2017, 118, 98-105.

54. W. Yang, W. Yang, F. Ding, L. Sang, Z. Ma and G. Shao, Carbon, 2017, 111, 419-427.
55. K. Fic, G. Lota, M. Meller and E. Frackowiak, Energy Environ. Sci., 2012, 5, $5842-5850$.

56. B. Liu, Y. Liu, H. Chen, M. Yang and H. Li, J. Power Sources, 2017, 341, 309-317.

57. J. Yi, Y. Qing, C. Wu, Y. Zeng, Y. Wu, X. Lu and Y. Tong, J. Power Sources, 2017, 351, 130-137. 